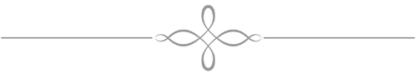

\section{Agroecologia: base da sustentabilidade dos agroecossistemas}

Daiane Loreto Vargas*

Andréia Furtado Fontoura**

José Geraldo Wizniewsky*

Resumo: Diante da insustentabilidade socioambiental dos sistemas de produção agrícolas e do modo de vida na atualidade, o enfoque agroecológico caracteriza-se como uma alternativa que trás a perspectiva de sustentabilidade aos agroecossistemas. A ciência agroecológica trabalha com a idéia de várias dimensões e com a questão da preservação da biodiversidade, da interação de diversas plantas e animais, onde deve-se manter uma diversificação de culturas, assim como a matéria orgânica e os microorganismos atuando no solo. Cenário que contribuirá para o aumento da produtividade, para o reestabelecimento do equilíbrio dos agroecossistemas e para a transformação ambiental e social de uma forma que se sustente ao longo do tempo.

\section{Agroecology: basis of sustainability agroecosystems}

Abstract: Given the environmental unsustainability of production systems and agricultural way of life today, the agroecological approach is characterized as an alternative that brings the perspective of sustainability to agroecosystems. The agroecological science works with the idea of various sizes and with the issue of biodiversity conservation, the interaction of various plants and animals, which must maintain a diversity of cultures, as well as organic matter and soil microorganisms acting. Scenario that will contribute to increased productivity for the reestablishment of the balance of agroecosystems and the environmental and social transformation in a way that sustains over time.
* Tecnóloga em Agropecuária - UERGS. Mestre em Extensão Rural - UFSM. Grupo de Pesquisa Extensão Rural Aplicada

**Engenheira Agronoma, Mestranda em Extensão Rural, Extensionista da Emater/RS.

*** Doutor em Agroecologia Sociologia e Estudos Campesinos, Professor Adjunto da Universidade Federal de Santa Maria, Departamento de Educação

Palavras-chave:

Agroecologia; Agroecossistemas; Sustentabilidade

Key-Words:

Agroecology; Agroecosystems; Sustainability 


\section{Introdução}

A sociedade moderna coloca suas necessidades de consumo e, por conseguinte, as demandas econômicas e de mercado assina de questões igualmente importantes, como as ações ambientais, sociais, éticas e culturais. Nesse sentido, o que se percebe nos estudos, teorias desenvolvidas através de pesquisas e na realidade empírica vivenciada no dia-a-dia, é o fato de que ultrapassamos todos os limites de crescimento. Percebe-se isso, a partir do momento em que o poder de regeneração dos recursos naturais não se mantém na mesma proporção em que são retirados os componentes do sistema. A partir dessa constatação, alguns autores colocam que o mundo não suportará por muito tempo o crescimento econômico baseado no atual padrão de extração e consumo dos recursos naturais (GOODLAND, 1997).

Este panorama coloca para a sociedade atual um cenário de insustentabilidade sob vários aspectos, dentre os mais preocupantes estão às questões sociais e ambientais. Surgindo a necessidade de pesquisas e exposição de experiências alternativas a esse "modelo de desenvolvimento". O qual tem por base 0 auto grau de consumo de energia, sem pensar na manutenção da vida das gerações futuras. Sendo assim, a valorização e a conservação da qualidade dos agroecossistemas tornam-se fundamental, através dos princípios de manutenção da biodiversidade natural do sistema, de forma a incentivar o equilíbrio ecológico entre as espécies e a sustentabilidade da produção agrícola.

Dessa forma, as vertentes teóricas que preconizam a idéia de agroecossistemas sustentáveis têm deixado de ser analisadas como uma visão teórico-científica, muitas vezes compreendida como utópica. A necessidade de se construir sistemas de produção sustentáveis é real, visível para a sociedade, seja através das catástrofes naturais, das conseqüências do aquecimento global, da degradação dos solos, da contaminação de mananciais, da pertinente pobreza e exclusão social. Cenário que preconiza a necessidade de mudanças consciente e que abre alas para conhecimento que, não são novos, mas, tem conotação de certa forma recente para a sociedade em geral, especialmente para aqueles que diretamente estão envolvidos com a agricultura.

Nesse ínterim, a agroecologia se apresenta como prática capaz de preservar e reconstruir sistemas de produção degradados pela ação do homem, no momento em que tem por base um pilar de seis dimensões básicas, as quais preconizam um re-pensar no próprio modo de vida. Dessa forma, a agroecologia corresponde a um campo de estudo que compreende o manejo ecológico dos recursos naturais, para que, através de uma ação social coletiva de caráter participativo, de um enfoque holístico e de uma estratégia sistêmica seja construído um modelo de agricultura e de vida sustentável.

Os pilares fundamentais dessa prática, que também é uma ciência, estão embasados nas dimensões sociais, ambientais, econômicas, culturais, éticas e políticas. Para entender toda essa complexidade, a partir dessa parte introdutória, o trabalho a seguir se dividi em três partes: a) agroecossitemas sustentáveis: uma necessidade frente a atual insustentabilidade socioambiental; b) agroecologia: entendida como ciência; c) agroecologia e os agroecossistemas sustentáveis. Tendo metodologicamente a base na pesquisa bibliográfica.

\section{Agroecossitemas sustentáveis: uma necessidade frente a atual insustentabilidade socioambiental}

Geografia Ensino \& Pesquisa, v. 17, n.1 p. 173-180, jan./abr. 2013

Agroecologia: base da sustentabilidade dos agroecossistemas
O sistema capitalista embasado na economia de mercado transformou a sociedade sob vários aspectos, como por exemplo, aqueles relacionados a cultura, as relações com o meio ambiente, as relações sociais e ao próprio conceito do que é realmente necessário para a vida humana no planeta. Essas questões ficam bastante claras quando começamos a observar os novos hábitos da vida social e as conseqüências dos mesmos sobre a questão ambiental e social.

A sociedade atual explora os recursos naturais para produzir alimentos (sistemas agrícolas) e bens materiais de consumo, sem se preocupar com o poder de regeneração dos mesmos, os 
quais não se mantêm ou não se regeneram na mesma proporção em que são retirados/ extraídos do sistema. Sendo assim, a sociedade ultrapassou os limites do crescimento para satisfazer uma série de necessidades criadas pelo homem moderno, pelo sistema capitalista e sua economia de mercado.

De acordo com Goodland (1997) a atividade humana vem contribuindo para a degradação da camada de ozônio e para o aumento da radiação ultravioleta, ocasionando maior incidência do câncer de pele e a redução da eficácia do sistema imunológico, dentre outras anormalidades. Além do aquecimento global, aumento da temperatura média em função do alto consumo de dióxido de carbono liberado pelo petróleo e pelo gás natural, todas essas questões estão ocasionado a intensidade de fenômenos naturais que antes eram raros.

Esses fenômenos, por sua vez, têm conseqüências nos vários segmentos da vida em sociedade, como na agricultura, na segurança alimentar e nos sistemas produtivos de uma forma geral. Implicando na produtividade das culturas, salinização, desertificação e erosão do solo. Ainda no final dos anos 90 , já haviam sido degradados $35 \%$ do solo terrestre. Seguindo o pensamento do autor supracitado, a economia humana consumia nos anos 90 , na forma de beneficiamento de "coisas" diversas para o seu consumo, $40 \%$ da produção primária de fotossíntese terrestre, contribuindo fortemente para a contaminação dos rios, desigualdade social e ambiental.

Uma preocupação dos autores nesse sentido é quanto a extinção de espécies, segundo Guzmàn (2009), desaparece anualmente em torno de 5.000 espécies, devido principalmente a invasão do seu habitat natural. A utilização da ciência e de novas tecnologias impôs um novo manejo dos recursos naturais, substituindo o conhecimento local e com isso a natureza passou a ser dominada pelo homem, o qual visando o lucro e a conquista de mercados começou a substituir o manejo dos recursos naturais e a produção de base ecológica pelos processos biológicos.

\section{Agroecologia: entendida como ciência}

Diante do cenário de insustentabilidade socioambiental apresentado anteriormente, nasce a nível mundial um consenso pela necessidade de novas estratégias, que contribuam para a construção de agroecossistemas sustentáveis, visando a segurança na produção de alimentos e a preservação ambiental (ALTIERI E NICHOLLS, 2000). Nesse sentido, tem-se o importante apoio da ciência agroecológica nos modos de produção agrícolas.

A muito se discute sobre o conceito de agroecologia, surgindo ao longo do tempo algumas correntes teóricas que divergem sobre o tema. A discussão das diferentes visões sobre a temática não é o objetivo desse trabalho, mas, é importante que se faça essa ressalva, para demonstrar que a abordagem a ser adotada neste estudo não é um consenso universal. Adota-se aqui, o conceito de agroecologia como o de uma ciência, um enfoque com bases científicas, que tem por objetivo dar sustentação para a "transição dos atuais modelos de desenvolvimento rural e de agricultura convencionais, para estilos de desenvolvimento rural e de agriculturas sustentáveis" (CAPORAL E COSTABEBER, 2002, p.4).

$O$ conceito adotado por Caporal e Costabeber (2004) segue o viés adotado por Miguel Altieri, um dos principais pesquisadores sobre a temática, os autores colocam que o entendimento do que é verdadeiramente a agroecologia surgiu da busca pela construção de novos conhecimentos:

[...] nasceu a Agroecologia, como um novo enfoque científico, capaz de dar suporte a uma transição a estilos de agriculturas sustentáveis e, portanto, contribuir para o estabelecimento de processos de desenvolvimento rural sustentável. A partir dos princípios ensinados pela Agroecologia passaria a ser estabelecido um novo caminho para a construção de agriculturas de base ecológica ou sustentáveis, como veremos adiante (CAPORAL E COSTABEBER, 2004, p.8).

Geografia Ensino \& Pesquisa, v. 17, n.1 p. 173-180, jan./abr. 2013

Vargas, D. L.; Fontoura, A. F.; Wizniewsky, J. G. 
Sob essa perspectiva, a agroecologia deixa de ser entendida como uma simples forma de intervenção diferenciada nos sistemas de produção agrícolas, passando a ser compreendida como um conhecimento que tem por base um método científico, baseando-se nos princípios da ecologia (GLIESSMAN, 2003). Nesse sentido, a ciência agroecológica não deve ser reduzida a um simples conjunto de técnicas agronômicas a serem aplicadas na agricultura, porque é um "enfoque científico, capaz de oferecer as ferramentas para a comparação entre diferentes formas de produção e suas respectivas lógicas de reprodução social e de apropriação da natureza" (CAPORAL E COSTABEBER, 2002, p. 13).

Metodologicamente, o enfoque agroecológico parte dos termos sociais da unidade de produção, seja "das comunidades, dos grupos, das cooperativas ou de outras formas de cooperação e sociabilização em que estejam organizados os agricultores" (CAPORAL E COSTABEBER, 2002, p. 21). Essa ciência tem como um dos seus princípios analisar as múltiplas dimensões do agroecossitema e as suas interações, rompendo com a visão difusionista e estabelecendo um método plural de análise e intervenção (CARDOSO, 2008). Pode ser entendida como uma vertente da ciência que trabalha com a idéia de sistemas, considerando os agroecossistemas e as interações que ocorrem nos mesmos, onde valoriza-se a diversidade e os saberes locais (VARGAS, et al. 2010).

Todo esse contexto corrobora com as questões levantadas por Costabeber e Caporal (2003), quando os mesmos colocam que é preciso considerar o processo social envolvido na prática agrícola. Reforçando a idéia de cidadania, onde se pensa a perspectiva do desenvolvimento rural sustentável com equidade entre todos os sujeitos envolvidos. Principalmente em termos de oportunidades a eles estendidas, buscando gradativamente e continuamente avanços nas seis dimensões da sustentabilidade, até que se consiga um equilíbrio entre os elementos no sistema.

Continuando com a visão dos autores, a agroecologia se aplica através dos processos da ecologia e dos processos sociais, partindo de um caráter participativo, onde se visa reconduzir a coevolução social e ecológica, mediante um controle das forças produtivas da natureza e da sociedade. Nesse sentido, o conhecimento local contribui fortemente para as estratégias de desenvolvimento, onde os saberes endógenos facilitam a construção de estilos de agricultura potencializadores da biodiversidade ecológica e da diversidade sociocultural.

\section{Agroecologia e os agroecossistemas sustentáveis}

A ciência agroecológica adota uma perspectiva ecológica nos processos de produção, onde a forma de produção é entendida como uma questão complexa que envolve vários fatores. Dentre estes, destaca-se a importância da produção agrícola com a preservação da biodiversidade de espécies, onde as interações entre pessoas, cultivos, solos e animais é fundamental para que se obtenha o equilíbrio do sistema. Na concepção de Gliessman (2003), deve-se ter consciência de que cada ecossistema tem uma capacidade de produção, e ainda, as bases do enfoque agroecológico visam manter a produtividade agrícola, mantendo a capacidade produtiva do solo, a qualidade e a quantidade dos alimentos ao longo prazo.

O enfoque agroecológico analisa os ecossistemas agrícolas como as principais unidades de estudos, suas particularidades e as interações que ocorrem com as mesmas são fundamentais nos estudos dentro da agroecologia. Nesse sentido, consideram-se as interações que ocorrem nos sistemas de produção, os ciclos minerais, as transformações da energia, dos processos biológicos e as relações socioeconômicas (ALTIERI E NICHOLLS, 2000).

A prática agrícola com base ecológica contribui para o restabelecimento do balanço energético de determinado sistema, através do manejo sustentável dos sistemas de produção e da preservação da biodiversidade natural. Pois, com a diversidade de espécies atuando num mesmo local, ocorrerão interações entre os componentes do sistema, onde alguns elementos serão utilizados para a produção de outros. Como exemplos podem ser citados: ervas daninhas utilizadas como forragem, esterco utilizado como fertilizante, dentre outros. 
De acordo Altieri e Nicholls (2000), são as interações entre os diversos componentes bióticos do agroecossistema que vão contribuir de forma positiva para o controle biológico de pragas, reciclagem de nutrientes, conservação da água, conservação e/ou regeneração do solo, além do aumento da produtividade agrícola de forma sustentável. Desde que se entendam as relações entre os componentes: solo, microorganismos, plantas, insetos herbívoros e inimigos naturais.

Dessa forma, na visão dos autores dentre os elementos básicos para que se construa um agroecossistema que se sustente a longo prazo, estão a conservação dos recursos renováveis, a adaptação dos cultivos ao meio ambiente e a diversificação de espécies animais e vegetais, porque é através dela que se realizam as interações necessárias para manter um solo produtivo.

Cultivar diversas espécies de forma integrada, fazendo uso de culturas anuais e permanentes, da rotação de culturas, incorporando num mesmo sistema árvores frutíferas e florestais, ou seja, plantas com diversidade genética, se possível arranjar com a produção de animais no mesmo sistema, contribuirá para a sustentabilidade daquele agroecossistema. Na Figura-1 estão arranjados os componentes citados pelos autores como fundamentais ao equilíbrio do agroecossistema.

Figura 1 - Componentes, funções e estratégias de melhoramento da biodiversidade nos agroecosistemas, adaptada de Altieri e Nicholls (2000).

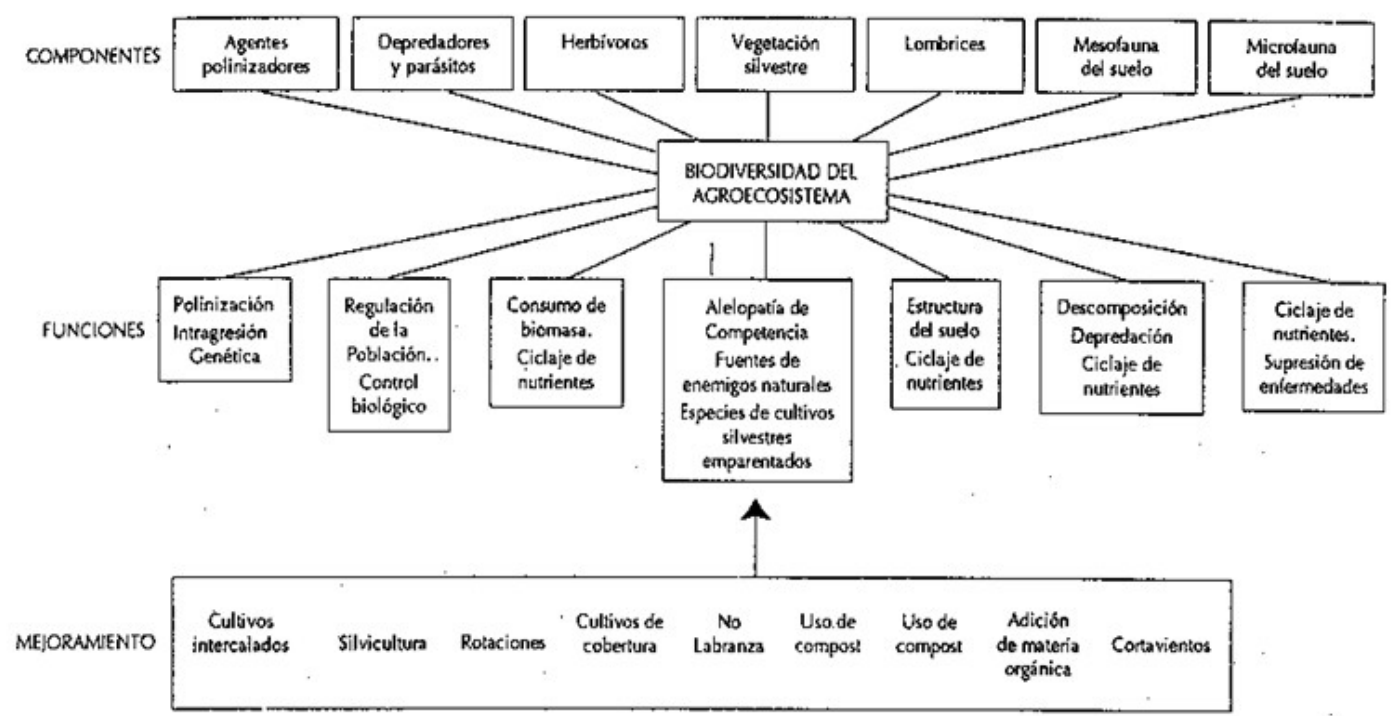

Como pode ser observado na Figura-1, Altieri e Nicholls (2000) destacam que existem fatores complementares a questão da diversidade de plantas e animais convivendo num mesmo sistema. Deve-se procurar fazer uso de cultivares com raízes diferenciadas em termos de profundidade, incorporar plantas com raízes profundas para recuperar e reciclar os nutrientes do solo, favorecer um bom microclima no ambiente. Além de, favorecer a produção de biomassa para estrumes, uma boa cobertura do solo para conservar a água, os nutrientes do próprio solo e a circulação dos microorganismos benéficos, através da manutenção da matéria-orgânica.

Essa biodiversidade nos sistemas de produção agrícolas deve ser manejadas através da rotação de culturas, utilização de cultivos de cobertura, cultivos intercalados, dentre outros, podendo ser utilizados cultivos anuais e perenes. Podem ser construídas ainda, barreiras de cata-vento, cinturões de proteção e cercas vivas, os quais vão melhorar o habitat dos microorganismos e insetos (ex: abelhas polinizadoras). Essas são algumas práticas importantes destacadas pelos autores para o manejo sustentável dos sistemas de produção.

Na visão de Gliessman (2003), a introdução de várias espécies num sistema de produção, a rotação de cultivos, e o fato de permitir a biodiversidade vão deixar que o sistema resista aos

Geografia Ensino \& Pesquisa, v. 17, n.1 p. 173-180, jan./abr. 2013

Vargas, D. L.; Fontoura, A. F.; Wizniewsky, J. G. 
problemas, porque serão delineados agroecossistemas com flexibilidade, resistência e capacidade de manter-se através do tempo. 0 autor trabalha com a idéia de que o processo de transição agroecológica possui um auto grau de complexidade e maior será a complexidade do sistema, quanto mais perfeito se queira chegar aos níveis de sustentabilidade.

Para Gliessman (2000) pode-se distinguir três níveis fundamentais no processo de transição ou conversão para agroecossistemas sustentáveis, utilizando-se os princípios da agroecologia. 0 primeiro nível, seria o início da mudança de sistema, se refere ao incremento e a eficiência das práticas convencionais para reduzir o uso e o consumo de insumos externos, os quais são escassos e daninhos ao meio ambiente, essa etapa tem como principal objetivo reduzir os impactos negativos ao ecossistema.

No segundo nível das fases de transição, trabalha-se com a substituição de insumos e práticas convencionais por práticas alternativas. Tendo como objetivo principal a substituição de insumos e práticas intensivas com produtos que contaminam e degradam o meio ambiente, por outras mais brandas sob o ponto de vista ecológico. Analisando o resultado dessa etapa, poderia se visualizar uma estrutura básica do agroecossistema pouco alterada.

Por fim, o terceiro nível na escala dos agroecossistemas sustentáveis e também o mais complexos de todos, de acordo com Gliessman (2000), seria o redesenho dos agroecossistemas. Nesse redesenho o sistema passara a funcionar com base em um conjunto de novos processos ecológicos. Essa etapa procura acabar com as causas dos problemas que não foram resolvidos nos dois níveis anteriores. Para entender os resultados práticos desses processos têm sido realizadas várias pesquisas em relação à transição do primeiro para o segundo nível, mas, os trabalhos que analisam o terceiro nível são poucos, e estes são muito interessantes de serem divulgados para que fosse possível visualizar os estilos de agriculturas sustentáveis.

\section{Conclusão}

Diante do que foi exposto até aqui, percebe-se que existem alternativas viáveis para uma produção alimentar sustentável no campo e para o equilíbrio socioambiental dos agroecossistemas. Essas alternativas passam pelos princípios e pelas práticas da agroecologia, onde a preservação da biodiversidade natural no campo trona-se imprescindível. A interação de várias espécies, os cuidados de manejo e a permanência de predadores naturais, são alguns fatores apontados pelos autores que contribuem o equilíbrio ecológico e para uma sinergia nos agroecossistemas, o que garante a sustentabilidade dos mesmos.

Vale lembrar que este é um processo de transformação do ambiente, onde é necessário tempo para a mudança, consciência da sociedade, aprendizado e envolvimento das pessoas, das famílias e/ou das comunidades. Pois, quando falamos em agroecologia estamos tratando de estratégias e conhecimentos que vão além de aspectos tecnológicos ou agronômicos da produção. Sendo necessário incorporar dimensões mais amplas e complexas, incluindo variáveis econômicas, sociais, ambientais, culturais, políticas e éticas da sustentabilidade.

\section{Referências Bibliográficas}

Geografia Ensino \& Pesquisa, v. 17, n.1 p. 173-180, jan./abr. 2013

\footnotetext{
Agroecologia: base da sustentabilidade dos agroecossistemas
}

ALTIERI, M; NICHOLLS, C, I. Agroecología: Teoría y práctica para uma agricultura sustentable. Série Textos Básicos para la Formación Ambiental. $1^{\text {a }}$ Edición. México: PNUMA, 2000, p.250. 
CAPORAL, F. R; COSTABEBER, J. A. Agroecologia enfoque científico e estratégico para apoiar o desenvolvimento rural sustentável (Texto provisório para debate). Porto Alegre: EMATER/RS-ASCAR, Junho de 2002. Disponível em: <http://www.agroecologia.uema.br/ publicacoes/AgroecolTexto.pdf>. Acesso: 17 de fev. 2010.

CAPORAL, F. R; COSTABEBER, J. A. Agroecologia: alguns conceitos e princípios/ 24 p. Brasília: MDA/SAF/DATER-IICA, 2004.

CARDOSO, J. H. Ecologia e outras dimensões dos agroecossistemas. Texto elaborado para o Seminário de Formação em Agroecologia, GATS, UFSM. 2008. Disponível em: <gats. synthasite.com>. Acesso: 23 de fev. 2012.

COSTABEBER, J. A; CAPORAL, F. R. Possibilidades e alternativas do desenvolvimento rural sustentável. In: VELA, H (Org.): Agricultura Familiar e Desenvolvimento Rural Sustentável no Mercosul. Santa Maria: Editora da UFSM/Pallotti, 2003. p.157-194.

GLIESSMAN, S. "A agricultura pode ser sustentável”. EMATER/RS. Rio Grande do Sul, 2003. Entrevista concedida a Jornalista Ângela Filippi. Disponível em: <http://www.emater.tche.br/ docs/agroeco/revista/n3/03-entrevista.htm>. Acesso: 21 de fev.de 2012.

GLIESSMAN, S. R. Agroecologia: processos ecológicos em agricultura sustentável. Porto Alegre: Editora da Universidade - UFRGS, 2000

GOODLAND, R. La Tesis de que el Mundo está en sus Límites. Medio ambiente y desarrollo sostenible : más allá del informe Brundtland. p. 19 -36, 1997. Disponível em: <http://dialnet.unirioja.es/servlet/articulo?codigo=576942>. Acesso: 04 de fev. de 2010.

GUZMÁN, E, S. Agroecología y desarrollo rural sustentable: una propuesta desde Latino América. 2009. Disponível em: <http://geografiaposgrado.files.wordpress. com/2009/04/agroecologiaydesarrollorura1.pdf>. Acesso em: 07 de fev. de 2010.

VARGAS, D. L; WIZNIEWSKY, J. G; HILLIG, C. O desenvolvimento rural sustentável alicerçado pela ciência agroecológica e pelo pronaf-agroecologia. In: Anais... Congresso Internacional de Responsabilidade e Sustentabilidade Socioambinetal, Foz do Iguaçu/ PR, 2010. Disponível em: http://www.isapg.com.br/2011/cirss/. Acesso: 20 de fev. 2012.

\section{Correspondência:}

Daiane Loreto Vargas- Rua: Padre João Bosco Penido Burnier, $n^{0} 742$, apto 101. Santa Maria, Rio Grande do Sul, Brasil

E-mail: loretodevargas@gmail.com

Recebido em 08 de abril de 2012.

Revisado pelo autor em 14 de janeiro de 2013.

Aceito para publicação em 17 de janeiro de 2013.

Geografia Ensino \& Pesquisa, v. 17, n.1 p. 173-180, jan./abr. 2013

Vargas, D. L.; Fontoura, A. F.; Wizniewsky, J. G. 